\title{
Introduction to the "Scoliosis" Journal Brace Technology Thematic Series: increasing existing knowledge and promoting future developments
}

\author{
Stefano Negrini ${ }^{1 * \dagger}$, Theodoros B Grivas ${ }^{2+}$
}

\begin{abstract}
Bracing is the main non-surgical intervention in the treatment of idiopathic scoliosis during growth, in hyperkyphosis (and Scheuermann disease) and occasionally for spondylolisthesis; it can be used in adult scoliosis, in the elderly when pathological curves lead to a forward leaning posture or in adults after traumatic injuries. Bracing can be defined as the application of external corrective forces to the trunk; rigid supports or elastic bands can be used and braces can be custom-made or prefabricated. The state of research in the field of conservative treatment is insufficient and while it can be stated that there is some evidence to support bracing, we must also acknowledge that today we do not have a common and generally accepted knowledge base, and that instead, individual expertise still prevails, giving rise to different schools of thought on brace construction and principles of correction. The only way to improve the knowledge and understanding of brace type and brace function is to establish a single and comprehensive source of information about bracing. This is what the Scoliosis Journal is going to do through the "Brace Technology" Thematic Series, where technical papers coming from the different schools will be published.
\end{abstract}

\section{Editorial}

Bracing is the main non-surgical intervention in the treatment of progressive idiopathic scoliosis (IS) during growth, sometimes applied with a specific exercise program [1-8]. Bracing can also be used in adult scoliosis $[9,10]$. During growth, braces are also prescribed to patients with hyperkyphosis (HK) (and Scheuermanns disease) [11-17] and occasionally for spondylolisthesis [18-23]. And finally, they are applied in the elderly when pathological curves lead to a forward leaning posture $[17,24,25]$ or in adults after traumatic injuries [26,27].

\section{Definition, goals of treatment and mechanisms of action}

Bracing can be defined as the application of external corrective forces to the trunk with the following goals:

- during growth, to halt curve progression or to correct pathological spinal curves [28-31];

\footnotetext{
* Correspondence: stefano.negrini@isico.it

+ Contributed equally

${ }^{1}$ ISICO (Italian Scientific Spine Institute), Via R Bellarmino 13/1, 20141 Milan, Italy
}

(c) 2010 Negrini and Grivas; licensee BioMed Central Ltd. This is an Open Access article distributed under the terms of the Creative Commons Attribution License (http://creativecommons.org/licenses/by/2.0), which permits unrestricted use, distribution, and reproduction in any medium, provided the original work is properly cited. collapse of already established pathological curves;

- after trauma, to allow proper vertebral healing and avoid progressive deformity [32,33].

To achieve these goals, rigid supports or elastic bands can be used [34,35] and braces can be custom-made or prefabricated [36-39].

Understanding the biomechanical action of a brace is of particular importance. The theoretical background for spinal correction is that the application of mechanical forces that reduce the pathological compression on given parts of the vertebral column while increasing it on others will result in a more symmetrical and natural loading that will, according to the literature:

- facilitate proper spinal growth [40-42],

- avoid progressive degeneration of the spine $[40,43,44]$,

- unload damaged vertebral segments and allow proper remodelling [32,33].

Although this is an old concept, the theory has been reinforced over time and in the field of IS was recently summarized in the "vicious cycle" hypothesis [44], where it is proposed that lateral spinal curvature 
produces asymmetrical loading of the skeletally immature spine through movement and neuromuscular control, which in turn causes asymmetrical growth and hence progressive wedging deformity. In this respect, the role of the intervertebral disks in the progression of IS and in its possible correction using bracing has also recently been considered $[45,46]$. Conversely, bracing could establish a useful "virtuous cycle", and as a result could lead to gradual reduction of the asymmetry present in scoliosis $[29,47]$. In accordance with these theories, a novel concept describing a comprehensive model of IS progression, based on the patho-biomechanics of the deforming "three joint complex" was also recently presented [48]. All these theories are also relevant in treating $\mathrm{HK}$ and the traumatic spine with a brace.

An alternative hypothesis suggests that the use of braces leads to neuro-motor reorganization caused by the changes in external and proprioceptive inputs and movement resulting from the constraint of bracing [49-52]. According to this hypothesis, braces are considered the drivers of movement while they increase external and internal bodily sensations. This permanently changes motor behaviours, even when the brace is removed, and can have a long-term effect on bone formation. Also, this hypothesis can be easily applied at all pathologies and ages; this hypothesis can be considered true in terms of trunk behaviour and neuro-muscular organization, while its possible effect on growing bone needs further investigation.

Two other interesting and significant concepts to explain the actions of the brace have been discussed. One suggests that the brace provides mechanical support to the body (passive component), while the other suggests that the patient pulls his/her body away from pressure sites (active component) to correct the curve. Such divergent theories illustrate the complexity of this problem, but the most important point of brace treatment is to provide the three dimensional correction of the spinal deformity, and methodologies must be developed with this in mind [53].

\section{Expertise and actual Evidence Based Clinical Practice}

The state of research in the field of conservative treatment is insufficient [54]. Interest in this specific topic decreased gradually from the 1970's to the 1990's, and only in the last decade has it improved, due to the efforts of the international scientific Society on Scoliosis Orthopedic and Rehabilitation Treatment (SOSORT) and its Journal (Scoliosis). What is clearly evident is that our understanding of brace effectiveness is still in its early stages of development. Braces are still named according to the town where it was developed [55-60] or the name of its inventor $[47,61,62]$ or its theory [63-66]. No actual classification system exists to help distinguish one brace from another [65,67], and only very few comparisons among different braces have been published [68-70]. The only way to improve the knowledge and understanding of brace type and brace function is to establish a single and comprehensive source of information about bracing. This is what the Scoliosis Journal is going to do. A study of each brace type is clearly the first step toward an understanding of the common roots and the specific differences among them. This will, hopefully, stimulate even more research on bracing in the future. Let us start by documenting the knowledge of our brace experts, and then use this to derive useful commonalities and increase our general knowledge.

Brace effectiveness is questioned by some clinicians and researchers because there is not enough evidence published to support it [71-73]. A Cochrane Review on the topic in Adolescent IS has been published in the January, 2010 issue of the Cochrane Database [31]. It concludes that there is evidence in support of bracing, but much of it is of very low quality. There are even fewer published papers on HK brace treatment $[15,16]$, and almost none exist in the treatment of spondylolisthesis [19]. While a few brace studies have been published in adults and in the elderly [25-27,32,33], a lot of research still needs to be done. Nevertheless, the existing results provide only weak evidence in favour of bracing in these clinical situations, and there is not a consensus that has been reached. Consequently, while it can be stated that there is evidence to support bracing, we must also acknowledge that today we do not have a common and generally accepted knowledge base, and that instead, individual expertise still prevails, giving rise to different schools of thought. In this respect, conservative experts have joined together in SOSORT, conceding that they may not share the same concept of the biomechanical action of corrective bracing on spinal curvature [29] even though they all agree on how to manage conservatively to obtain good results [74]. The first step must be to combine our collective knowledge and hold it up to scrutiny, so that a careful and thorough investigation of each theory can be completed.

We must also develop clear, consistent definitions of all the parameters used to measure brace effectivenes, because without this it will be impossible to compare the effectiveness of different brace types and the relative performance of the different centers involved. To help accomplish this, SOSORT is organizing its next consensus paper on this topic. It will be discussed at the 2010 Montreal SOSORT meeting and then published in Scoliosis. This will be an important first step 
in evaluating the effectiveness of bracing. Recently, the standardization of criteria for Adolescent IS brace research has been established by the Scoliosis Research Society (SRS) Committee on Bracing and Non-operative Management [75,76]. The application of these criteria will greatly enhance research protocols exploring the effectiveness of bracing, and it is anticipated that much progress will be made in the near future as a consequence [31,77-80].

\section{Other issues in bracing}

\section{The issue of compliance}

In many studies, compliance with brace wear is measured by asking patients if they used their brace and how many hours of wear they had each day [81]. Some researchers added to this by looking for signs of wear on the brace to determine whether this matched with the patients report [82]. Some studies have reported that the amount of strap tension was highly correlated with the in-brace correction and the treatment outcomes [83]. Such variability in the way that compliance is measured has prompted the International Research Society on Spinal Deformities (IRSSD) to develop new tools to measure compliance more objectively. Recent improvement in electronics technology have given us new ways to accurately measure brace wear, and this is making research much more reliable. Some devices use temperature or humidity sensors for measuring purposes while others use force switches and pressure sensors [53].

\section{The issue of pressure being applied}

How do we define optimal brace tightness? The in-brace correction depends on curve flexibility (which correlates highly with treatment outcomes) and the amount of pressure that the brace exerts. The optimal amount of pressure may be different in each patient. Most researchers have only recorded how much time the brace has been worn and do not record (or are unable to record) whether the brace has been worn correctly, especially in terms of the amount of pressure being applied. This is unfortunate because the absence of force measurement may result in a distorted view of the overall compliance [53].

\section{The issue of treatment time}

Another important issue is the number of hours that a brace should be worn by the patient on a daily basis. Unfortunately, guidance given to the patient is generally based on 'clinical intuition'. The most commonly recommended treatment time is 23 hours per day. In recent years, the SRS has raised doubts as to whether part-time brace wearing is effective at all. If part-time treatment is effective then the question arises: How many hours per day is sufficient? There is no doubt that prediction of brace treatment outcome is difficult, but it becomes more so when treatment time is not standardized and we cannot accurately determine the risk of progression, the in-brace correction, or the compliance (both wear time and wear tightness) [53].

The issue of bending radiographs

What is the best way to determine the flexibility of the spine? Although bending radiographs can provide accurate flexibility information, exposing growing children to additional radiation is undesirable [53]. Should radiographic data be used in scoliosis research on brace effectiveness and if yes, which way?

\section{Characteristics of articles published in the "Brace Technology" Thematic Series of the Journal}

The articles published in the "Brace Technology" Thematic Series of the ScoliosisJournal will comply with some essential characteristics and format. Manuscripts submitted for the series should be written on braces whose efficacy has been confirmed either in the very short term (at least six months), short term (end of growth) or long term (follow up beyond growth). All custom made or pre-fabricated corrective braces for spinal deformity (including non-idiopathic scoliosis, HK, Scheuermann's disease, spondylolisthesis, etc.) in all ages can be submitted and published in this section.

The "Brace Technology" articles describing specific braces should be organized as follows:

- Introduction: With general notes and goals of the study

- History: A short history of the brace

- Theoretical principles: How the brace might work (theory). General description of the mechanical principles of correction, the classification used for prescription, and the variations of the brace according to the curve pattern

- The brace: A description of the brace including photos of the front, back and sides in as many as possible curve types according to the classification used for prescription

- Practical issues. This should be divided in the following parts:

-How to prescribe the brace: principles of correction written in prescriptions by MDs

-How to build the brace: principles of construction by CPOs, with some photos; (see also the discussion regarding brace pressure above)

-How to check the brace: principles of checking by MDs and CPOs

-Protocols: description of the protocols generally used according to each clinical situations; (see criteria for bracing)

-Everyday usage: the number of hours per day that the patient will wear the brace 
-Exercises: specific exercises while in the brace (if any), with photos of how to perform them

- Results \& case reports: Short review of published results. The results should be divided into groups related to braces whose efficacy has been confirmed in the very short term (at least 6 months), short term (end of growth) or long term (follow-up beyond growth). Also, the type of study performed (case series, controlled) and the population considered must be reported. Moreover, 1 to 3 cases (of different curve types) fully documented with photos, clinical data and $x$-rays should be reported. However, patient consent must be obtained for this data and provided upon submission.

- Discussion: comparison with other braces based on the author's hypothesis; strengths and limitations, advantages and disadvantages

- Conclusions: with final remarks

- The abstract should be organized with the following sections: background, brace description and principles, results, conclusions

Moreover, in this thematic series, technical notes concerning particular details of brace construction could also be invited and published, to increase awareness and understanding of bracing. These articles can include theoretical comparison among braces, classification proposals, indications for future research directions, technical notes concerning particular details of brace construction, devices developed to accompany braces, and so on.

\section{Conclusion}

Scoliosis journal is focused on spinal deformity. Even though there is some early evidence in favour of bracing [31], the actual knowledge in the field does not yet allow us to classify the existing braces and categorize them beyond the names proposed by the original authors [65,67]. Consequently, the only possible way to increase our collective knowledge in the field is to publish what is being done today by clinicians with the most expertise in a systematic way, so to allow progressive comparisons and a deeper understanding. Moreover, discussion must be open among these experts, and these contributions will be accepted and published in this same thematic series of the journal. We are confident that with this new effort the journal will become an important source of information to the world of spinal deformity management, and will increase our understanding of how bracing effects the outcome of these problems. We do all this for the benefit of our patients.

\section{Acknowledgements}

We express our thanks to Drs Tomasz Kotwicki, Manuel Rigo and Jean Claude de Mauroy for reading the text and for their advice. We also thank Dr. Patrick Knott for copy-editing this article.

\section{Author details}

'ISICO (Italian Scientific Spine Institute), Via R Bellarmino 13/1, 20141 Milan, Italy. ${ }^{2}$ Orthopaedic and Trauma Department, "Tzanio" General Hospital of Piraeus, Piraeus, Greece.

\section{Authors' contributions}

SN and TBG contributed equally in the manuscript drafting. The authors read and approved the final manuscript.

\section{Competing interests}

The authors declare that they have no competing interests.

Received: 26 January 2010

Accepted: 28 January 2010 Published: 28 January 2010

\section{References}

1. Rowe DE, Bernstein SM, Riddick MF, Adler F, Emans JB, Gardner-Bonneau D: A meta-analysis of the efficacy of non-operative treatments for idiopathic scoliosis. J Bone Joint Surg Am 1997, 79:664-674.

2. Negrini S, Antonini G, Carabalona R, Minozzi S: Physical exercises as a treatment for adolescent idiopathic scoliosis. A systematic review. Pediat Rehabil 2003, 6(3-4):227-235.

3. Romano M, Negrini S: Manual therapy as a conservative treatment for adolescent idiopathic scoliosis: a systematic review. Scoliosis 2008, 3:2.

4. Negrini S, Fusco C, Minozzi S, Atanasio S, Zaina F, Romano M: Exercises reduce the progression rate of adolescent idiopathic scoliosis: results of a comprehensive systematic review of the literature. Disabil Rehabil 2008, 30(10):772-785.

5. Lenssinck ML, Frijlink AC, Berger MY, Bierman-Zeinstra SM, Verkerk K, Verhagen AP: Effect of bracing and other conservative interventions in the treatment of idiopathic scoliosis in adolescents: a systematic review of clinical trials. Phys Ther 2005, 85(12):1329-1339.

6. Weiss HR, Negrini S, Rigo M, Kotwicki T, Hawes MC, Grivas TB, Maruyama T, Landauer F: Indications for conservative management of scoliosis (guidelines). Scoliosis 2006, 1(1):5

7. Weiss HR, Negrini S, Hawes MC, Rigo M, Kotwicki T, Grivas TB, Maruyama T: Physical exercises in the treatment of idiopathic scoliosis at risk of brace treatment - SOSORT consensus paper 2005. Scoliosis 2006, 1:6.

8. Rigo M, Negrini S, Weiss HR, Grivas TB, Maruyama T, Kotwicki T: SOSORT consensus paper on brace action: TLSO biomechanics of correction (investigating the rationale for force vector selection). Scoliosis 2006, 1:11.

9. Winter RB, Lonstein JE: Adult scoliosis. Instr Course Lect 1983, 32:170-191.

10. Weiss HR, Dallmayer R: Brace treatment of spinal claudication in an adult with lumbar scoliosis-a case report. Stud Health Technol Inform 2006, 123:586-589.

11. de Mauroy JC: Standards of management of juvenile \& adolescent Kyphosis with corrective braces and physiotherapy in everyday clinics. 4th SOSORT Meeting: 2009; Lyon 2009.

12. Pizzutillo PD: Nonsurgical treatment of kyphosis. Instr Course Lect 2004 53:485-491.

13. Riddle EC, Bowen JR, Shah SA, Moran EF, Lawall H Jr: The duPont kyphosis brace for the treatment of adolescent Scheuermann kyphosis. J South Orthop Assoc 2003, 12(3):135-140.

14. Wenger DR, Frick SL: Scheuermann kyphosis. Spine 1999, 24(24):2630-2639.

15. Weiss HR, Turnbull D, Bohr S: Brace treatment for patients with Scheuermann's disease - a review of the literature and first experiences with a new brace design. Scoliosis 2009, 4:22

16. Zaina F, Atanasio S, Ferraro C, Fusco C, Negrini A, Romano M, Negrini S: Review of rehabilitation and orthopedic conservative approach to sagittal plane diseases during growth: hyperkyphosis, junctional kyphosis, and Scheuermann disease. Eur J Phys Rehabil Med 2009, 45(4):595-603

17. Negrini S: Focus on flexed posture and hyperkyphosis: prevention and rehabilitation to reduce disability and increase quality of life. Eur J Phys Rehabil Med 2009, 45(4):567-569. 
18. Weiss HR, Dallmayer R: Brace treatment of spinal claudication in an adolescent with a grade IV spondylosisthesis-a case report. Stud Health Technol Inform 2006, 123:590-593.

19. Negrini S, Monticone M, Paroli C: Efficacy of antilordotic TLSO braces to reduce spondylolisthesis in adolescents: preliminary results from a clinical retrospective study. 1st International Conference on Conservative Management of Spinal Deformities: 23-24 January 2004 2004; Barcelona: SOSORT (Study Group on Orthopaedic and Rehabilitation Treament) 2004.

20. Negrini S, Sibilla P: Efficacy of antilordotic TLSO braces to reduce spondylolisthesis in adolescents: a clinical retrospective study. Scoliosis Research Society Annual Meeting: September 2001 2001; Vancouver: Scoliosis Research Society 2001.

21. Lonstein JE: Spondylolisthesis in children. Cause, natural history, and management. Spine 1999, 24(24):2640-2648.

22. Blanda J, Bethem D, Moats W, Lew M: Defects of pars interarticularis in athletes: a protocol for nonoperative treatment. J Spinal Disord 1993, 6(5):406-411.

23. Pizzutillo PD, Hummer CD: Nonoperative treatment for painful adolescent spondylolysis or spondylolisthesis. J Pediatr Orthop 1989, 9(5):538-540.

24. Weiss HR, Werkmann M: Treatment of chronic low back pain in patients with spinal deformities using a sagittal re-alignment brace. Scoliosis 2009, $4: 7$

25. Kado DM: The rehabilitation of hyperkyphotic posture in the elderly. Eur J Phys Rehabil Med 2009, 45(4):583-593.

26. Bailey CS, Dvorak MF, Thomas KC, Boyd MC, Paquett S, Kwon BK, France J, Gurr KR, Bailey SI, Fisher CG: Comparison of thoracolumbosacral orthosis and no orthosis for the treatment of thoracolumbar burst fractures: interim analysis of a multicenter randomized clinical equivalence trial. $J$ Neurosurg Spine 2009, 11(3):295-303.

27. Schmelzer-Schmied N, Cartens C, Meeder PJ, Dafonseca K: Comparison of kyphoplasty with use of a calcium phosphate cement and nonoperative therapy in patients with traumatic non-osteoporotic vertebral fractures. Eur Spine J 2009, 18(5):624-629.

28. The Scoliosis Research Society Brace Manual. Introduction. http://www. srs.org/professionals/bracing_manuals/section1.pdf.

29. Rigo M, Negrini S, Weiss H, Grivas T, Maruyama T, Kotwicki T: SOSORT consensus paper on brace action: TLSO biomechanics of correction (investigating the rationale for force vector selection). Scoliosis 2006, 1:11.

30. Grivas TB, Vasiliadis E, Chatziargiropoulos T, Polyzois VD, Gatos K: The effect of a modified Boston brace with anti-rotatory blades on the progression of curves in idiopathic scoliosis: aetiologic implications. Pediatr Rehabil 2003, 6(3-4):237-242

31. Negrini S, Minozzi S, Bettany-Saltikov J, Zaina F, Chockalingam N, Grivas TB, Kotwicki T, Maruyama T, Romano M, Vasiliadis ES: Braces for idiopathic scoliosis in adolescents. Cochrane Database Syst Rev 2010, 1: CD006850.

32. Stadhouder A, Buskens E, Vergroesen DA, Fidler MW, de Nies F, Oner FC: Nonoperative treatment of thoracic and lumbar spine fractures: a prospective randomized study of different treatment options. J Orthop Trauma 2009, 23(8):588-594.

33. Al-Khalifa FK, Adjei N, Yee AJ, Finkelstein JA: Patterns of collapse in thoracolumbar burst fractures. J Spinal Disord Tech 2005, 18(5):410-412.

34. Coillard C, Leroux MA, Zabjek KF, Rivard CH: SpineCor-a non-rigid brace for the treatment of idiopathic scoliosis: post-treatment results. Eur Spine J 2003, 12(2):141-148.

35. Wong MS, Cheng JC, Lam TP, Ng BK, Sin SW, Lee-Shum SL, Chow DH, Tam SY: The effect of rigid versus flexible spinal orthosis on the clinical efficacy and acceptance of the patients with adolescent idiopathic scoliosis. Spine 2008, 33(12):1360-1365.

36. Weiss HR, Rigo M: The cheneau concept of bracing-actual standards. Stud Health Technol Inform 2008, 135:291-302.

37. Sankar WN, Albrektson J, Lerman L, Tolo VT, Skaggs DL: Scoliosis in-brace curve correction and patient preference of CAD/CAM versus plaster molded TLSOs. J Child Orthop 2007, 1(6):345-349.

38. Wong MS, Cheng JC, Wong MW, So SF: A work study of the CAD/CAM method and conventional manual method in the fabrication of spinal orthoses for patients with adolescent idiopathic scoliosis. Prosthet Orthot Int 2005, 29(1):93-104.

39. Wong MS, Cheng JC, LO KH: A comparison of treatment effectiveness between the CAD/CAM method and the manual method for managing adolescent idiopathic scoliosis. Prosthet Orthot Int 2005, 29(1):105-111.
40. Lupparelli S, Pola E, Pitta L, Mazza O, De Santis V, Aulisa L: Biomechanical factors affecting progression of structural scoliotic curves of the spine. Stud Health Technol Inform 2002, 91:81-85.

41. Castro FP Jr: Adolescent idiopathic scoliosis, bracing, and the HueterVolkmann principle. Spine J 2003, 3(3):180-185.

42. Weiss HR, Hawes MC: Adolescent idiopathic scoliosis, bracing and the Hueter-Volkmann principle. Spine J 2004, 4(4):484-485.

43. Stokes IA: Mechanical modulation of spinal growth and progression of adolescent scoliosis. Stud Health Technol Inform 2008, 135:75-83.

44. Stokes IA, Burwell RG, Dangerfield PH: Biomechanical spinal growth modulation and progressive adolescent scoliosis - a test of the 'vicious cycle' pathogenetic hypothesis: Summary of an electronic focus group debate of the IBSE. Scoliosis 2006, 1:16.

45. Grivas TB, Vasiliadis E, Malakasis M, Mouzakis V, Segos D: Intervertebral disc biomechanics in the pathogenesis of idiopathic scoliosis. Stud Health Technol Inform 2006, 123:80-83.

46. Grivas TB, Vasiliadis ES, Rodopoulos G, Bardakos N: The role of the intervertebral disc in correction of scoliotic curves. A theoretical model of idiopathic scoliosis pathogenesis. Stud Health Technol Inform 2008, 140:33-36.

47. Rigo M, Weiss HR: The Cheneau concept of bracing-biomechanical aspects. Stud Health Technol Inform 2008, 135:303-319.

48. Grivas T, Vasiliadis ES, Triantafyllopoulos G, Kaspiris A: A comprehensive model of idiopathic scoliosis (IS) progression, based on the pathobiomechanics of the deforming "three joint complex". Scoliosis 2009, 4(Suppl 2):010.

49. Coillard C, Leroux MA, Badeaux J, Rivard CH: SPINECOR: a new therapeutic approach for idiopathic scoliosis. Stud Health Technol Inform 2002, 88:215-217.

50. Odermatt D, Mathieu PA, Beausejour M, Labelle H, Aubin CE: Electromyography of scoliotic patients treated with a brace. J Orthop Res 2003, 21(5):931-936

51. Negrini S, Marchini G, Tomaello L: The Sforzesco brace and SPoRT concept (Symmetric, Patient-oriented, Rigid, Three-dimensional) versus the Lyon brace and 3-point systems for bracing idiopathic scoliosis. Stud Health Technol Inform 2006, 123:245-249.

52. Smania N, Picelli A, Romano M, Negrini S: Neurophysiological basis of rehabilitation of adolescent idiopathic scoliosis. Disabil Rehabil 2008, 30(10):763-771.

53. Bagnall KM, Grivas TB, Alos N, Asher M, Aubin CE, Burwell RG Dangerfield PH, Edouard T, Hill D, Lou E, et al: The International Research Society of Spinal Deformities (IRSSD) and its contribution to science. Scoliosis 2009, 4(1):28.

54. Negrini S: Approach to scoliosis changed due to causes other than evidence: patients call for conservative (rehabilitation) experts to join in team orthopedic surgeons. Disabil Rehabil 2008, 30(10):731-741.

55. de Mauroy JC, Fender P, Tato B, Lusenti P, Ferracane G: Lyon brace. Stud Health Technol Inform 2008, 135:327-340.

56. Blount WP, Schmidt A: The Milwaukee brace in the treatment of scoliosis J Bone Joint Surg 1957, 37:693.

57. Watts HG, Hall JE, Stanish W: The Boston brace system for the treatment of low thoracic and lumbar scoliosis by the use of a girdle without superstructure. Clin Orthop Relat Res 1977, , 126: 87-92.

58. The Providence Scoliosis System. http://www.srs.org/professionals/ bracing_manuals/section8.pdf.

59. Bassett GS, Bunnell WP: Effect of a thoracolumbosacral orthosis on lateral trunk shift in idiopathic scoliosis. J Pediatr Orthop 1986, 6(2):182-185.

60. Katz DE, Richards BS, Browne RH, Herring JA: A comparison between the Boston brace and the Charleston bending brace in adolescent idiopathic scoliosis. Spine 1997, 22(12):1302-1312.

61. Cheneau J: Das Cheneau-Korsett. Ein Handbuch. Dortmund: Orthopadie Technik 1993, 1.

62. Risser JC: Scoliosis treated by cast correction and spine fusion. Clin Orthop Relat Res 1976, , 116: 86-94.

63. Weiss HR, Werkmann M, Stephan C: Correction effects of the ScoliOlogiC (R) "Cheneau light" brace in patients with scoliosis. Scoliosis 2007, 2(1):2.

64. Negrini S, Marchini G, Tomaello L: Efficacy of the Symmetric, Patientoriented, Rigid, Three-Dimensional (SPoRT) concept of bracing for scoliosis: a pair-controlled retrospettive short-term study on the Sforzesco Brace. 3rd International SOSORT Meeting: 2006 April 07-08 2006; Poznan, Poland: SOSORT 2006. 
65. Negrini S, Zaina F, Atanasio S: BRACE MAP, a proposal for a new classification of braces. Stud Health Technol Inform 2008, 140:299-302.

66. Sibilla P, Frassi A, Massimini M: II corsetto di Maguelone. 5 Congresso del Gruppo Italiano Scoliosi: 1982: GIS 1982.

67. Negrini S: Bracing adolescent idiopathic scoliosis today. Disabil Rehabil Assist Technol 2008, 3(3):107-111.

68. Negrini S, Marchini G: Efficacy of the symmetric, patient-oriented, rigid, three-dimensional, active (SPoRT) concept of bracing for scoliosis: a prospective study of the Sforzesco versus Lyon brace. Eura Medicophys 2007, 43(2):171-181, discussion 183-174.

69. Negrini S, Atanasio S, Negrini F, Zaina F, Marchini G: The Sforzesco brace can replace cast in the correction of adolescent idiopathic scoliosis: A controlled prospective cohort study. Scoliosis 2008, 3(1):15.

70. Weiss HR, Weiss GM: Brace treatment during pubertal growth spurt in girls with idiopathic scoliosis (IS): a prospective trial comparing two different concepts. Pediatr Rehabil 2005, 8(3):199-206.

71. Goldberg CJ, Dowling FE, Hall JE, Emans JB: A statistical comparison between natural history of idiopathic scoliosis and brace treatment in skeletally immature adolescent girls. Spine 1993, 18(7):902-908.

72. Dickson RA: Spinal deformity-adolescent idiopathic scoliosis. Nonoperative treatment. Spine 1999, 24(24):2601-2606.

73. Dickson RA, Weinstein SL: Bracing (and screening)-yes or no?. J Bone Joint Surg Br 1999, 81(2):193-198.

74. Negrini S, Grivas TB, Kotwicki T, Rigo M, Zaina F: Guidelines on "Standards of management of idiopathic scoliosis with corrective braces in everyday clinics and in clinical research": SOSORT Consensus 2008. Scoliosis 2009, 4(1):2

75. Thompson GH, Richards BS III: Inclusion and assessment criteria for conservative scoliosis treatment. Stud Health Technol Inform 2008, 135:157-163.

76. Richards BS, Bernstein RM, D'Amato CR, Thompson GH: Standardization of criteria for adolescent idiopathic scoliosis brace studies: SRS Committee on Bracing and Nonoperative Management. Spine 2005, 30(18):2068-2075, discussion 2076-2067.

77. Janicki JA, Poe-Kochert C, Armstrong DG, Thompson GH: A comparison of the thoracolumbosacral orthoses and providence orthosis in the treatment of adolescent idiopathic scoliosis: results using the new SRS inclusion and assessment criteria for bracing studies. J Pediatr Orthop 2007, 27(4):369-374.

78. Coillard C, Vachon V, Circo AB, Beausejour M, Rivard CH: Effectiveness of the SpineCor brace based on the new standardized criteria proposed by the scoliosis research society for adolescent idiopathic scoliosis. J Pediatr Orthop 2007, 27(4):375-379.

79. Aulisa AG, Guzzanti V, Galli M, Perisano C, Falciglia F, Aulisa L: Treatment of thoraco-lumbar curves in adolescent females affected by idiopathic scoliosis with a progressive action short brace (PASB): assessment of results according to the SRS committee on bracing and nonoperative management standardization criteria. Scoliosis 2009, 4:21.

80. Negrini S, Atanasio S, Fusco C, Zaina F: Effectiveness of complete conservative treatment for adolescent idiopathic scoliosis (bracing and exercises) based on SOSORT management criteria: results according to the SRS criteria for bracing studies - SOSORT Award 2009 Winner. Scoliosis 2009, 4:19.

81. Brace Wear Compliance. http://www.srs.org/professionals/ bracing_manuals/section3.pdf.

82. Landauer $\mathrm{F}$, Wimmer $\mathrm{C}$, Behensky $\mathrm{H}$ : Estimating the final outcome of brace treatment for idiopathic thoracic scoliosis at 6-month follow-up. Pediatr Rehabil 2003, 6(3-4):201-207.

83. Mac-Thiong JM, Petit Y, Aubin CE, Delorme S, Dansereau J, Labelle H: Biomechanical evaluation of the Boston brace system for the treatment of adolescent idiopathic scoliosis: relationship between strap tension and brace interface forces. Spine 2004, 29(1):26-32.

doi:10.1186/1748-7161-5-2

Cite this article as: Negrini and Grivas: Introduction to the "Scoliosis" Journal Brace Technology Thematic Series: increasing existing knowledge and promoting future developments. Scoliosis 2010 5:2.

\section{Submit your next manuscript to BioMed Central and take full advantage of:}

- Convenient online submission

- Thorough peer review

- No space constraints or color figure charges

- Immediate publication on acceptance

- Inclusion in PubMed, CAS, Scopus and Google Scholar

- Research which is freely available for redistribution

Submit your manuscript at www.biomedcentral.com/submit 